\title{
Study of acoustic characteristics of noise barriers
}

\author{
Valeriya Kulkina and Alexandr Komkin* \\ B M STU, Power Engineering Department, 105005, M oscow, Russia
}

\begin{abstract}
The acoustic efficiency of noise barriers has been studied using the developed two-dimensional finite element model in the COMSOL Multiphysics software package. Numerical calculations of the semi-infinite barrier efficiency have been compared with calculations conducted by the $M$ aekawa formula. The main attention has been paid to the influence of the underlying surface on barrier acoustic characteristics. The barrier acoustic efficiency depends on its height, the position of the noise source and on the calculation point above the underlying surface. This dependence has been presented in this research.
\end{abstract}

\section{Introduction}

A One of the main methods of protection against noise on the path from a sound source to a protected area is a noise barrier (acoustic barrier). Barriers differ in purpose, shape and material of manufacture. A fairly large number of publications both in Russia and abroad have been devoted to study noise barriers [1-14]. They usually use the optical-diffraction theory of acoustics with the use of Fresnel number. This number takes into account the difference in the path of sound beams in the presence and absence of an acoustic barrier.

In practice, calculating the efficiency of acoustic barriers is carried out using Maekawa formula [1-3], which is the result of the experimental data approximation. This approach of calculation is attractive since it is simple. However; it has its limitations as it is valid only for a semi-infinite absolutely rigid barrier. Therefore; it cannot take into account the presence of an underlying (reflective) surface on which the barrier is placed. In this case, the characteristics of the underlying surface and the barrier material cannot be taken into account. It is very difficult to take these factors into account when performing calculations using analytical methods. Although much attention is still paid to these issues [4-14], it should be borne in mind that in the near future numerical methods will become the main tool for calculating acoustic barriers, as well as in many other areas of applied acoustics [15-19]. In this paper, using finite element modeling, the features of the formation of a sound field near a barrier located on the underlying surface are studied in order to estimate the influence of this surface on the acoustic efficiency of the barrier.

* Corresponding author: akomkin@ mail.ru 


\section{Finite element model}

For studying the acoustic characteristics of noise barriers, linear acoustics methods have been used and simulations have been performed in the COMSOL Multiphysics software package. Figure 1 shows a two-dimensional finite element model. A circle with a diameter of 70 meters was constructed with a center at the beginning of the coordinate system, in which noise source (NS) was also located. The boundary conditions on the circumference bounding this area correspond to the characteristic impedance of air $Z=\rho c=415 \mathrm{~kg} / \mathrm{cm}^{2}$.

Non-uniform partitioning with a maximum finite element size of $0.01 \mathrm{~m}$ has been used in the simulation. Total number of finite elements is $17,578,135$. The calculations have been carried out at different frequencies. The upper frequency equals $4 \mathrm{kHz}$ which corresponds to a wavelength of $0.086 \mathrm{~m}$ and exceeds the maximum element size by almost an order of magnitude.

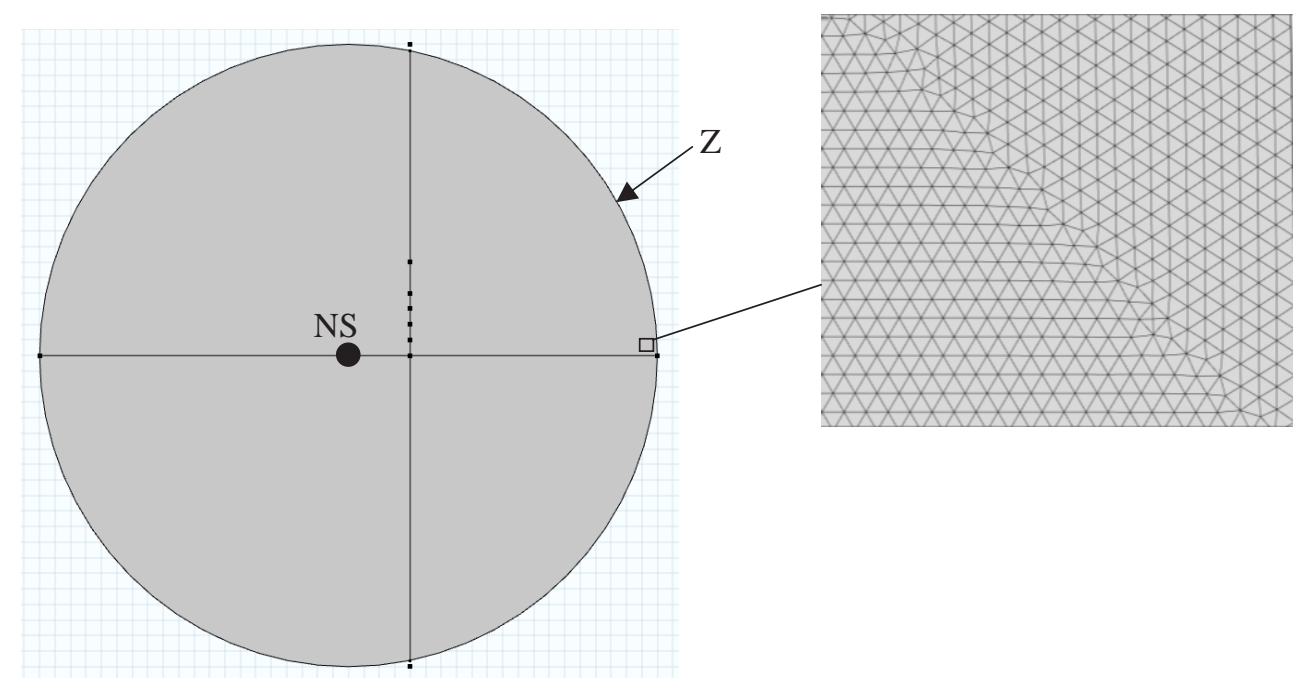

Fig. 1. Finite Element M odel and M esh Example.

At the first stage of the modeling, a semi-infinite barrier was considered. Here and further on, the barrier was located at a distance of 3 meters from the noise source. The distance from the upper edge of the barrier to the horizontal coordinate axis was considered as the effective height of the barrier he.

Figure 2 shows a picture of the sound pressure levels distribution in space on a frequency of $500 \mathrm{~Hz}$ for a model without a barrier and with a semi-infinite barrier with an effective height he $=2 \mathrm{~m}$. In the absence of a barrier, the picture corresponds to the propagating cylindrical waves from the center of coordinates. Presence of the barrier significantly complicates the sound field. It can be seen that standing waves are formed between the source and the barrier. In the space behind the barrier, the level of sound pressure significantly decreases. 


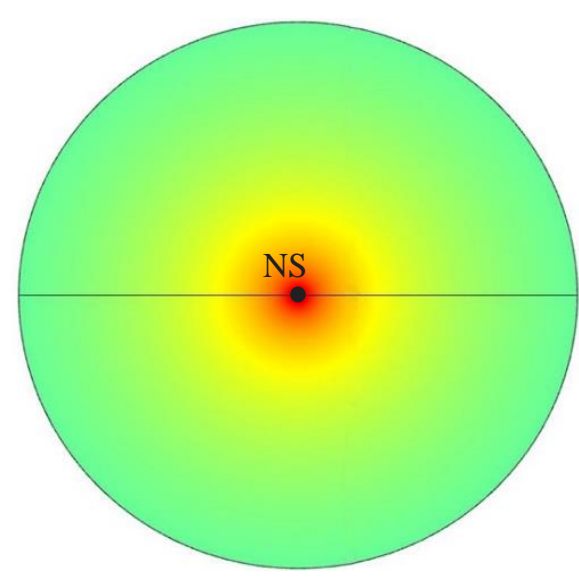

(a)

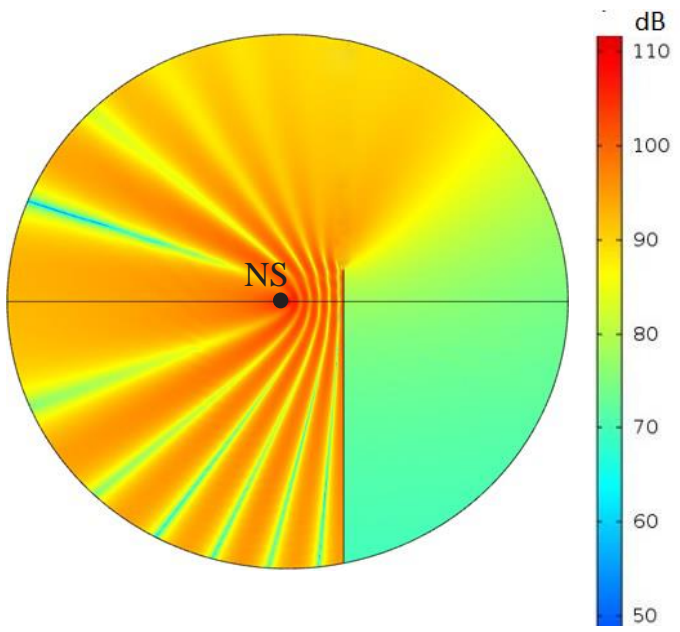

(b)

Fig. 2. Distribution of sound pressure levels in space for a model without a barrier (a) and with a semi-infinite barrier (b).

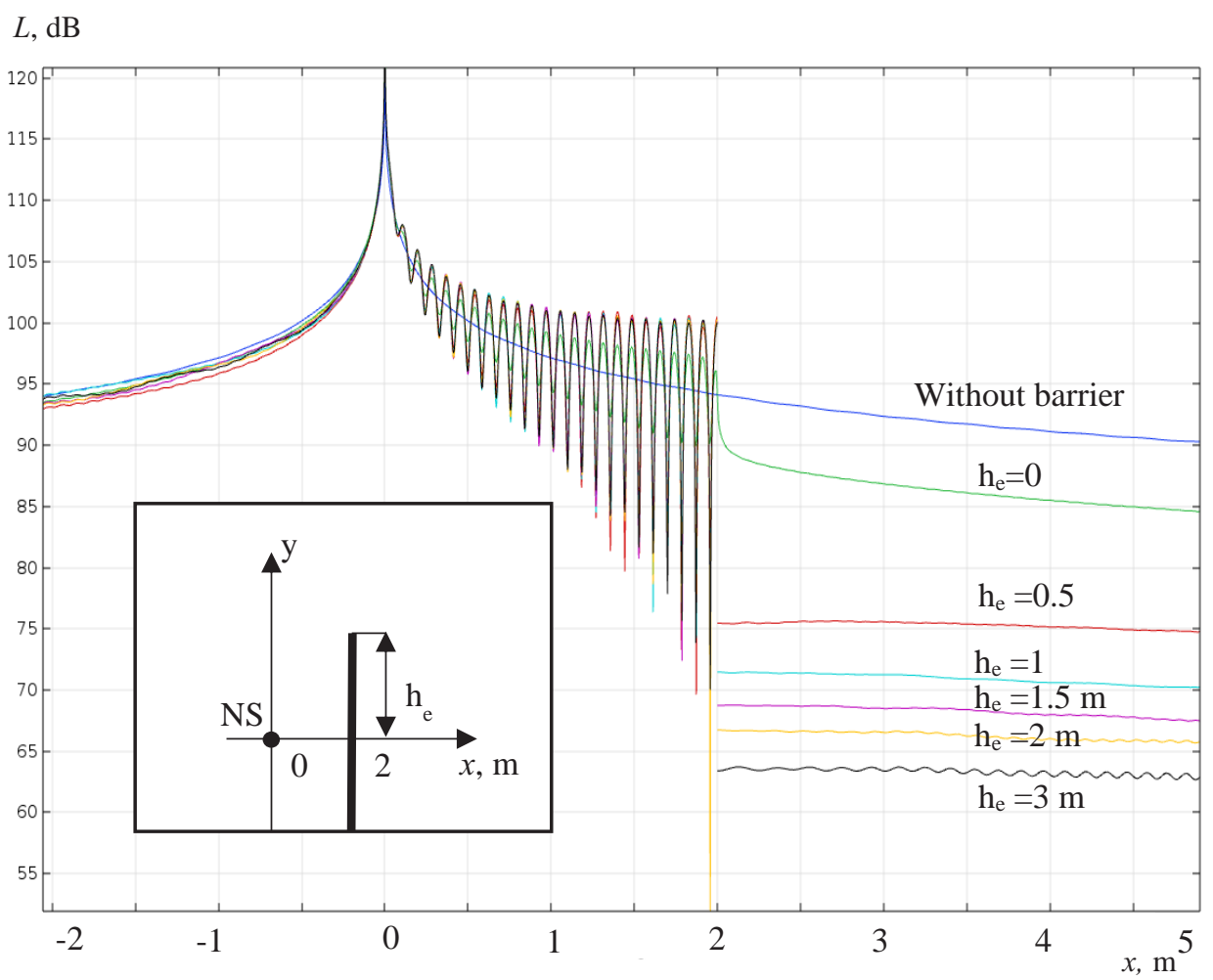

Fig. 3. Change in sound pressure levels al ong the transverse axis of the barrier at a frequency of $500 \mathrm{~Hz}$.

Figure 3 shows the change in sound pressure levels along the horizontal axis perpendicular to the plane of the barrier at a frequency of $500 \mathrm{~Hz}$. In the presented graphical dependencies, three areas can be distinguished. In the first area, to the left of the coordinates 
origin, there is a gradual decrease in sound pressure levels with distance from the source. In the second area, located to the right of the origin, but to the left of the barrier, there are oscillations of sound pressure levels with a period equal to the sound wavelength. These oscillations are due to the appearance of standing waves associated with the reflection of the incident sound wave from the barrier in this area. In the third area, located to the right of the barrier, there is a sharp drop in sound pressure levels followed by reaching a certain constant level. When the barrier height he increases, the value of this level decreases. At a barrier height of more than two meters, fluctuations in sound pressure become noticeable on the constant pressure curves. At the same time, the higher the barrier height is, the lower the corresponding constant sound pressure level is, and the more the noticeable fluctuations against its background are. Presence of such fluctuations is due to the appearance of weak standing waves due to the imperfection of the matched load.

A 1: 4 scale model was used to reduce errors in the calculation of the acoustic efficiency of high barriers, which are associated with small reflections from the boundaries due to the imperfection of the agreed load. This allows to limit the height of the barrier to $3 \mathrm{~m}$, which in reality corresponds to a $12 \mathrm{~m}$ barrier. Sound frequencies at which calculations were carried out also increased by 4 times. This made it possible to keep the value of Fresnel number unchanged and therefore, ensure the equivalence of the calculation values on the model and the value for full-sized barriers.

The main acoustic characteristic of the barrier is its acoustic efficiency

$$
\Delta L=L_{\text {with }}-L_{\text {without }}
$$

where $L_{\text {with }}$ and $\mathrm{L}_{\text {without }}$ are sound pressure levels at the control point without a barrier and with a barrier respectively.

Figure 4 shows the results of calculating the acoustic efficiency of barriers at a frequency of $125 \mathrm{~Hz}$, obtained by numerical and analytical methods (according to the Maekawa formula).

The general trend in the obtained dependence is a smooth increase in the efficiency of the barrier with an increase in its height. In addition, as expected, its effectiveness decreases with increasing distance from the control point to the barrier. This behavior is typical for the other considered frequency of $500 \mathrm{~Hz}$. The presented results show good agreement between numerical and analytical calculations. However, there are some differences that depend on the distance of the control point from the barrier. At a distance of $8 \mathrm{~m}$ analytical calculations give lower values than numerical ones. At a distance of $24 \mathrm{~m}$, starting from a barrier height of $6 \mathrm{~m}$ and higher, analytical calculations begin to give larger values than numerical ones.
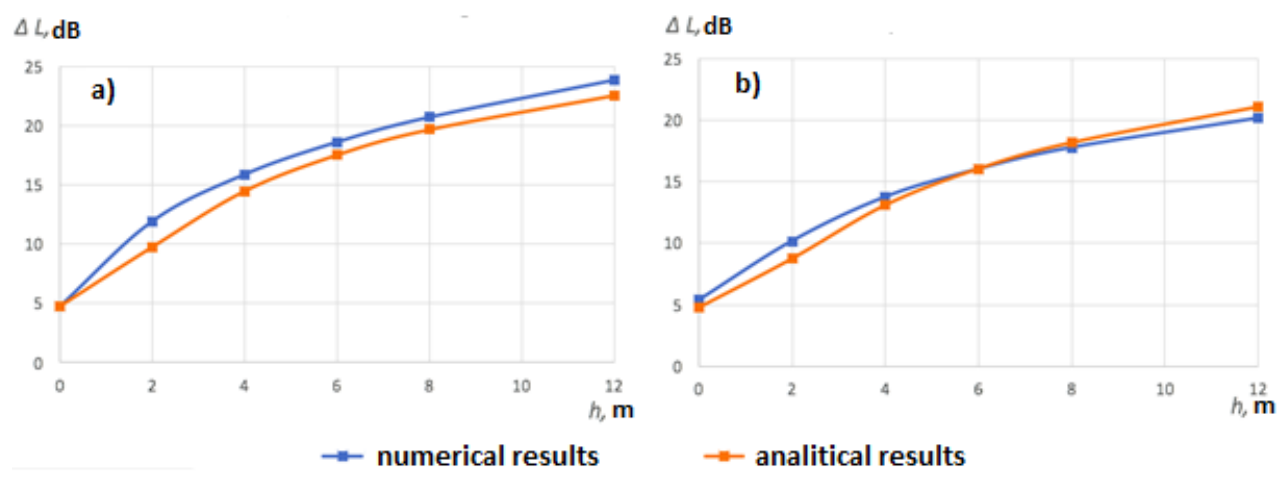

Fig. 4. A coustic efficiency of the barrier calculated by numerical and analytical methods for a frequency of $125 \mathrm{~Hz}$ for a distance from the control point to the barrier of $8 \mathrm{~m} \mathrm{(a)}$ and $24 \mathrm{~m}(\mathrm{~b})$. 
When the noise source is located above the reflecting surface, for example, on the rigid ground, the pattern of sound pressure distribution changes significantly, becoming more diverse (Figure 5,a). This is due to interference of the direct wave from the source and reflected from the surface. As a result, fanciful interference patterns are formed with periodically arranged beams of maxima and minima of sound pressure. As the frequency of sound increases, so does the number of such rays, which is seen in Figure 5,b.

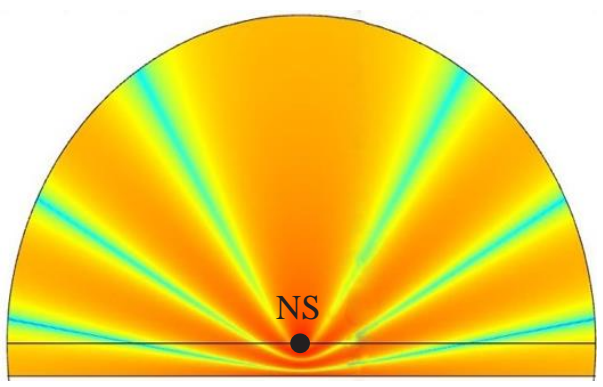

(a)

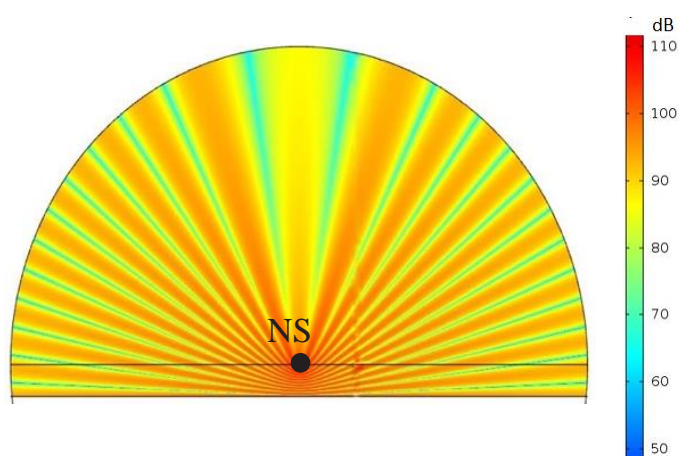

(b)

Fig. 5. Distribution of sound pressure levels over rigid ground with a source at a height $h_{1}=4 \mathrm{~m}$ at frequencies of $125 \mathrm{~Hz}$ (a) and $500 \mathrm{~Hz}(\mathrm{~b})$.

When the barrier is placed on a reflective surface, on the ground for example, the sound pressure distribution pattern changes significantly and becomes more diverse. This is due to the interference between the direct wave from the source and the reflected waves from the surface. As a result, bizarre interference patterns are formed with periodically arranged beams of maxima and minima of sound pressure. When sound frequency increases, number of such rays also increases. This pattern is observed in Figure 6.

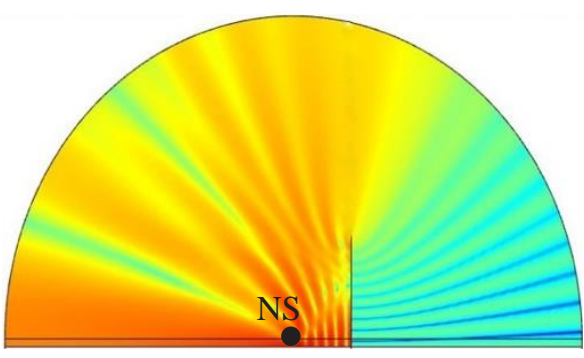

(a)

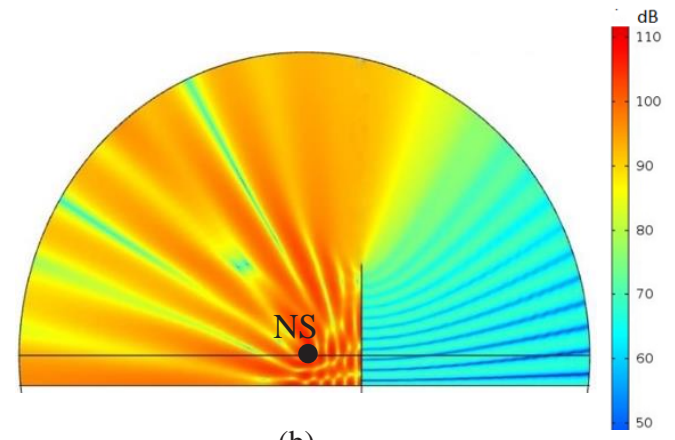

(b)

Fig. 6. The distribution of sound pressure levels over a rigid ground in the presence of the barrier with $h_{e}=12 \mathrm{~m}$ at a frequency of $125 \mathrm{~Hz}$ with source at $h_{1}=1 \mathrm{~m}(\mathrm{a})$ and $h_{1}=4 \mathrm{~m}$ (b)

Pattern of the sound pressure distribution depends both on the height of the sound source location, the height of the barrier, and the distance between the source and the barrier. The patterns depicted in Figure 6 show that the sound field behind the barrier is significantly reduced, however there is also an interference pattern in the form of rays of dark blue color. 
In addition, the acoustic efficiency of the barrier located above the reflecting surface significantly depends on the sound frequency, as shown by a comparison of Figures 6 and 7 .

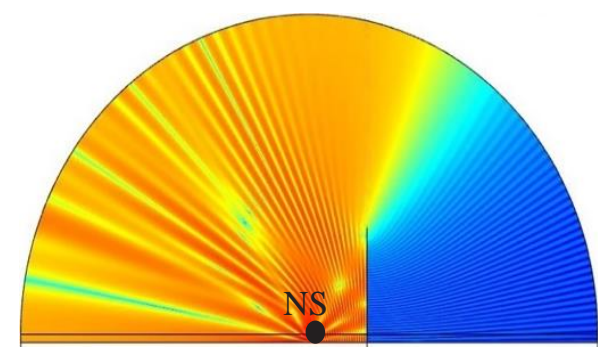

(a)

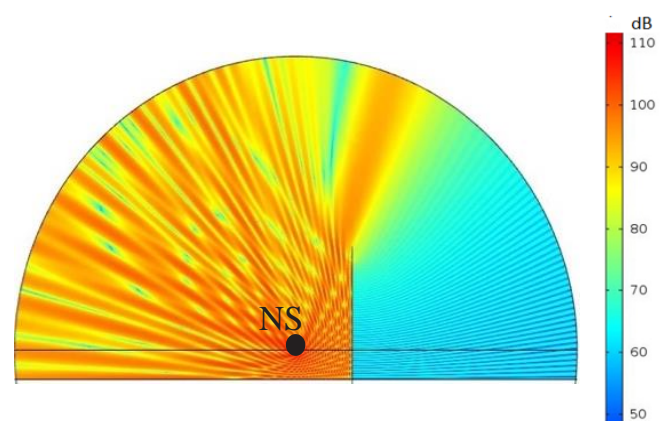

(b)

Fig. 7. The distribution of sound pressure levels over a rigid ground in the presence of the barrier with $h_{e}=12 \mathrm{~m}$ at a frequency of $500 \mathrm{~Hz}$ with source at $h_{1}=1 \mathrm{~m} \mathrm{(a)}$ and $h_{1}=4 \mathrm{~m}(\mathrm{~b})$.

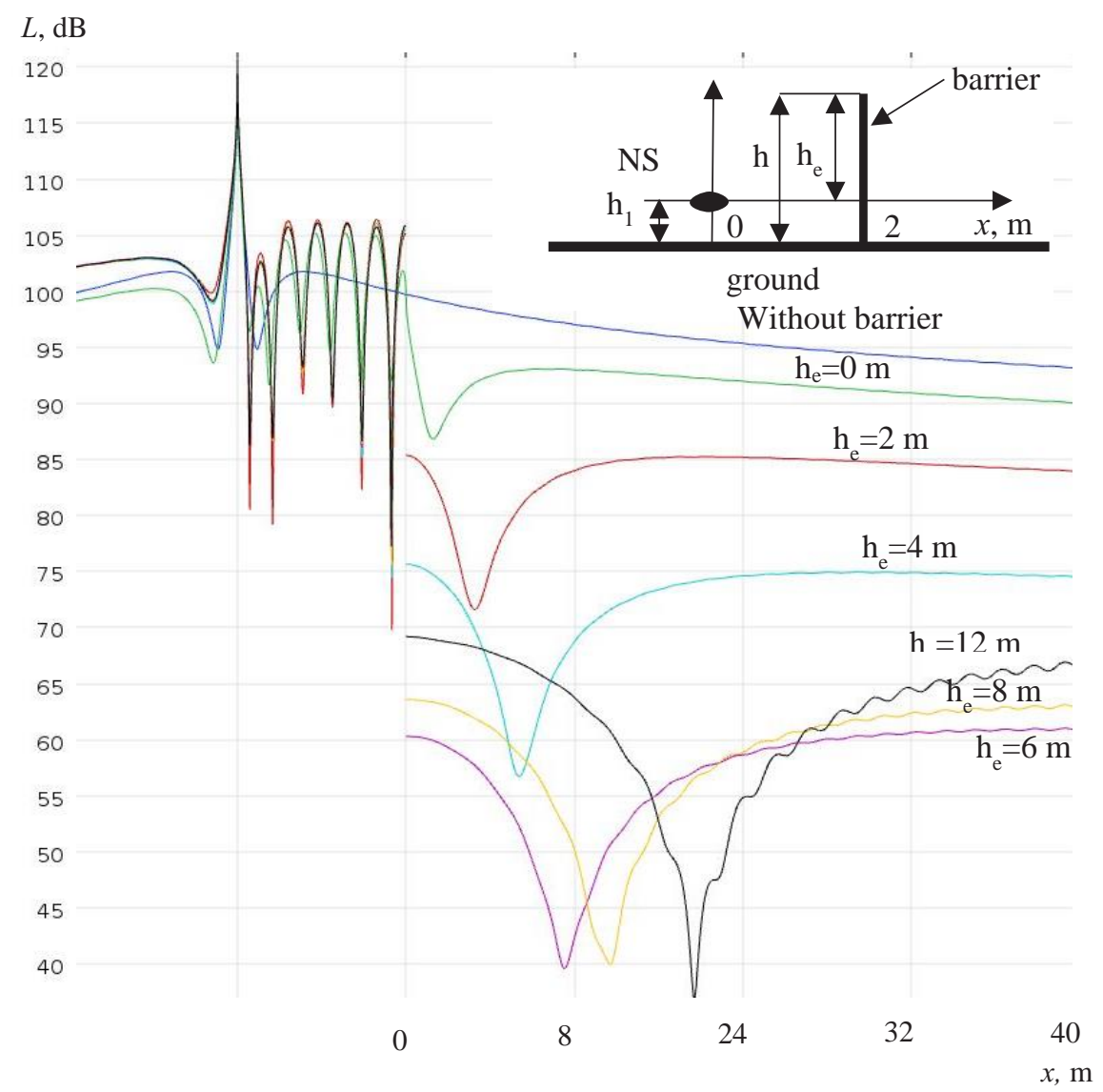

Fig.8. Change in sound pressure levels al ong the transverse axis of a barrier at a frequency of $125 \mathrm{~Hz}$ over a rigid ground. 
In order to identify the existing patterns when acoustic efficiency of the barrier changes in such a configuration (over the reflecting surface), the patterns of the sound pressure level distribution in the transverse plane, for different effective heights of the barrier, have been obtained as shown in Figure 8. Here and below, all calculations were carried out for a source with $\mathrm{h} 1=1 \mathrm{~m}$. It was noticed that for each barrier height there is its own distance to the control point, at which the minimum sound pressure (dips) is observed, which corresponds to its maximum efficiency. At the same time, the location of these dips does not have a direct relationship with the barrier height.

The dependences of the barrier efficiency on its height he as shown in Figure 9 for three control points allow us to conclude the following. In contrast to a semi-infinite barrier, the barrier efficiency does not increase smoothly with its height. This relationship is more complex, in which the maximum efficiency of the barrier does not correspond to its highest height, as was the case when there is no reflecting surface. Its position also depends on the frequency of the sound. Interestingly, this maximum barrier efficiency significantly exceeds the corresponding efficiency of a semi-infinite barrier.

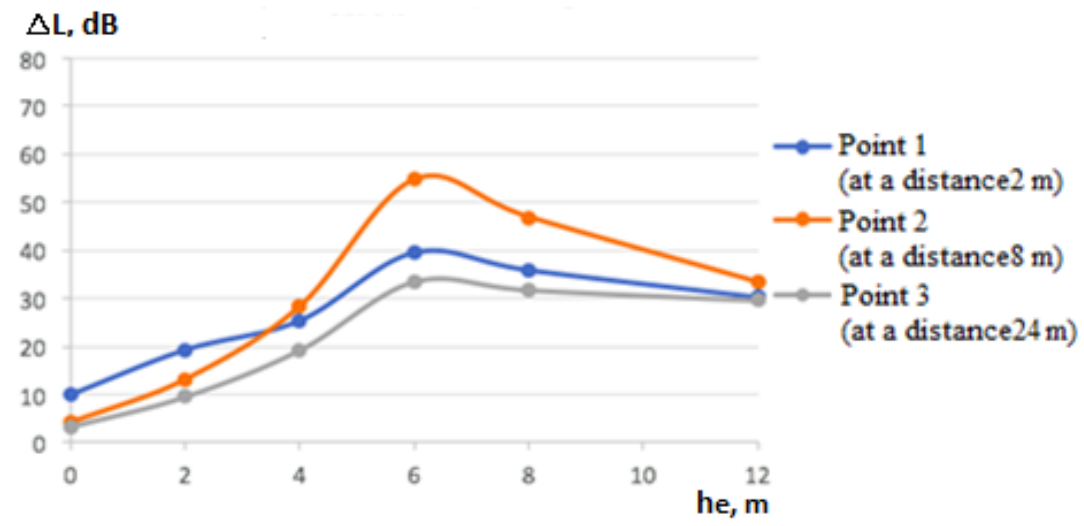

Fig.9. Dependences of the barrier acoustic efficiency on its height he at a frequency of $125 \mathrm{~Hz}$.

To understand the peculiarities of forming an acoustic pattern over the reflecting surface in the presence of a barrier, another model was studied, where the reflecting surface was located only behind the barrier. Patterns of sound pressure distribution were obtained at a frequency of $500 \mathrm{~Hz}$ in the vicinity of the barrier with he $=12 \mathrm{~m}$ without a reflecting surface (Figure 10,a), with a reflecting surface only behind the barrier (Figure10,b) and with a reflecting surface (Figure10,c).

From these patterns, it follows that at this frequency, the most effective barrier has a reflecting surface in front of and behind the barrier, while the color palette of the space behind the barrier corresponds to a low level of sound pressure, about $40 \mathrm{~dB}$. For a barrier without a reflecting surface, the corresponding sound pressure increases to about $60 \mathrm{~dB}$. The barrier with the reflecting surface behind it has the lowest acoustic efficiency when the sound pressure increases by about $10 \mathrm{~dB}$, reaching $70 \mathrm{~dB}$. 


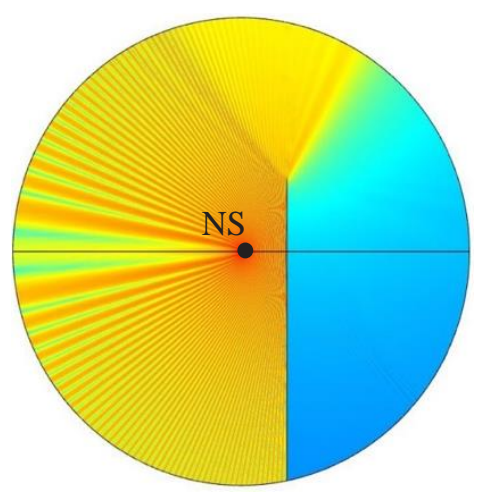

(a)

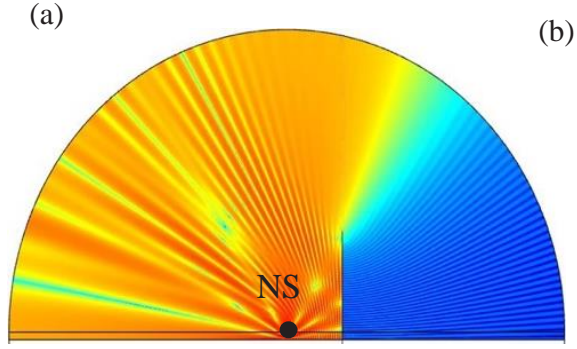

(c)

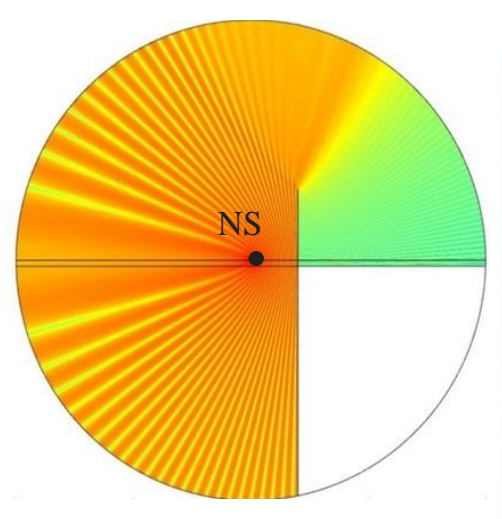

(b)

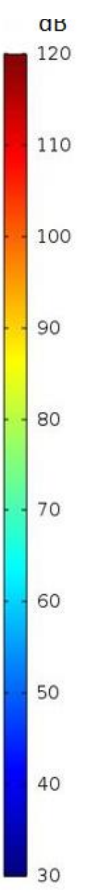

\section{as}

110

90

80

0

50

40

30

Fig.10. Sound pressure distribution patterns without a rigid ground (a), and with a rigid ground only behind the barrier (b) and a rigid ground in front and behind the barrier (c).

As before, the graphs of the sound pressure distribution have been obtained for the case of a reflective surface behind the barrier. In this case, it has been found that the coordinates of the sound pressure dips increase with increasing barrier height. The height dependences on acoustic barriers presented here also show that they have local intensity maxima. The position of this maxima, as before, depends on the sound frequency. A characteristic feature of the results obtained in this case is that the efficiency of the barrier is significantly lower than the efficiency of a barrier with a full reflecting surface. This is due to the presence of interference between the direct and the reflected waves in the area in front of the barrier. The result of these reflected waves is an attenuated sound wave which hits the upper edge of the barrier.

However, it should be borne in mind that such results are valid only for a given frequency of $125 \mathrm{~Hz}$. If we consider a different frequency, there may be a completely different picture. 


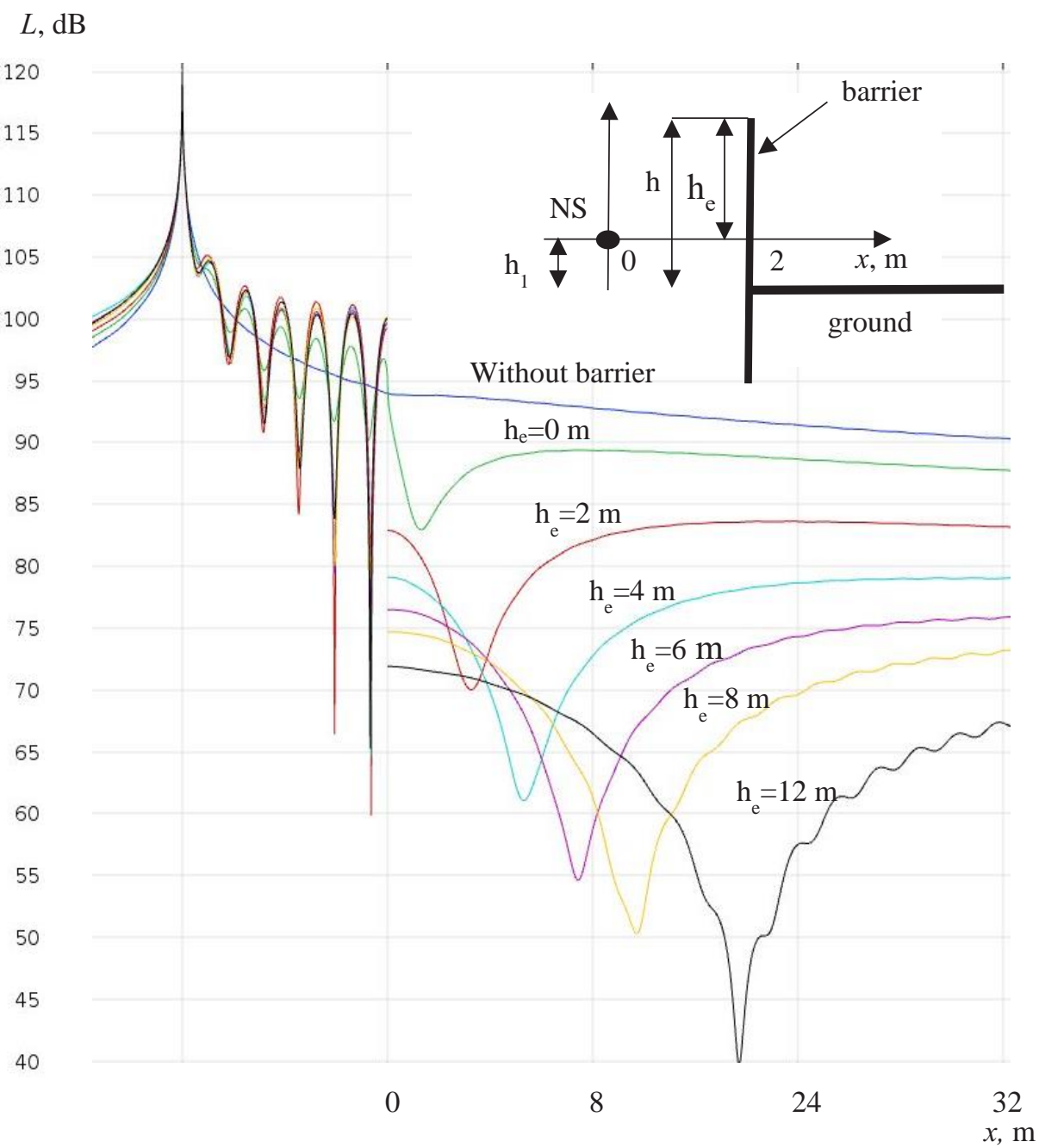

Fig. 12. Change in sound pressure levels along the transverse axis of the barrier in the presence of the barrier with a rigid ground behind the barrier at a frequency of $125 \mathrm{~Hz}$.

\section{Conclusion}

Studies have shown that the effectiveness of an acoustic barrier depends significantly on the presence of a reflective surface. At the same time, the maximum efficiency does not always correspond to the highest barrier height. This height of the barrier depends significantly on the frequency of sound.

It is proposed to conduct further numerical studies of barriers in order to obtain the integral efficiency of the barrier in dBA. In addition, there is a plan to conduct experimental studies on barriers in an acoustic chamber in order to confirm the obtained calculated results. 


\section{References}

1. Z. M aekawa, A ppl. A coust. 1, 157 (1968)

2. Z. M aekawa, Acoustic barriers, in $\mathrm{N}$ oise reduction in buildings and residential areas, Ed. by F.L. Osipov and E.Y a. Y udin. (Stroyizdat, M oscow, 1987) [in Russsian]

3. Z. M aekawa, Recent Problems with Noise Barriers, in Proceedings of the N oise-93 Conference, 31 M ay 31- 3 J une 1993, St. Petersburg, Russia (1993)

4. U. J. Kurze, J. A coust. Soc. A m. 55, 504 (1974)

5. T. Isei, T. F.W. Embleton, J. E. Piercy, J. A coust. Soc. A m. 67, 46 (1980)

6. A . L bsperance, J. Nicolas, G. A. Daigle, J. A coust. Soc. A m. 86, 1060 (1989)

7. S. I. Hayek, A ppl. A coust. 31, 77 (1990)

8. K. Takagi, A ppl. A coust. 31, 119 (1990)

9. Y. W. Lam, A ppl. A coust. 42, 29 (1994)

10. A. M uradaliand, K. R. Fyfe, A ppl. A coust. 53, 49 (1998)

11. P. M enounou, I. J. Busch-V ishniac, D. T. Blackstock, J. A coust. Soc. A m. 107, 2973 (2000)

12. P. M enounou, J. A coust. Soc. A m. 110, 1828 (2001)

13. P. M enounou, E. S. Papaef thymiou, A ppl. A coust. 71, 351 (2010)

14. G. Rosenhouse, The use of simplex approach for analysis of semi-infinite and finite sound barriers, in Proceedings of Inter-N oise 2019, 16-19 J une 2019, M adrid (2019)

15. A. I. Komkin, A. I. Bykov, A coust. Phys. 62(3), 269 (2016)

16. M. Kalugin et al., Her. Bauman M osc. St. Tech. Univ. Ser. Natur. Scien. 3, 32 (2018)

17. A. E pikhin, M. K raposhin, K. V atutin The numerical simulation of compressible jet at low Reynolds number using OpenFOAM, E3S W eb of Conferences. EDP Sciences, 128, 10008 (2019)

18. S. M. Sivachev, L. L. M yagkov Thermomechanical Fatigue Analysis of Diesel Engine Piston: Finite Element Simulation and Lifetime Prediction Technique, in Proceedings of International Conference on Industrial Engineering. Springer, Cham, 109 (2019)

19. Y. I. V inogradov, G. B. M enkov, Modeling of the boundary conditions when calculating the stress-strain state of a cone-shaped aircraft nose fairing, AIP Conference Proceedings, A IP Publishing LLC, 2171 (1) P. 170012 (2019) 